\section{Simulated models for testing performance of a radiofrequen- cy ablation device for spine tumors}

\author{
Federica Rusinà, ${ }^{1}$ Alice Ravizza, ${ }^{1}$ \\ Stefano Pasquino, ${ }^{2}$ Mario Muto $^{3}$ \\ 1USE-ME-D srl I3P Politecnico di \\ Torino, Torino, Italy; ${ }^{2}$ Myra Medical \\ SARL, Savigny, Switzerland; \\ 3Department of Neuroradiology, \\ A.O.R.N. Cardarelli, Naples, Italy
}

\begin{abstract}
Effectiveness of simulation models to assess performance a radiofrequency tumor ablation device.
\end{abstract}

\section{Introduction}

This study investigated and validated the use of different simulated vertebral models to test a device for radiofrequency thermal ablation of spine tumors (SPINERY, Axon $\mathrm{srl})$. SPINERY is a surgically invasive device (as the therapeutic treatment is administered using a RF needle inserted in the vertebral target area through surgical incision); design team assessed its operating principle in a preliminary set of functional simulated tests, to limit the use of animal models. The preliminary phase was a detailed evaluation of state-of-the-art about surgical acecesses, RF provision at local site and subsequent healing times. In the initial phase, different simulated models were selected: saline solution, bovine liver slices, a Becker full of egg albumen, and a 3D-printed vertebra model filled with bovine liver homogenate. ${ }^{1}$ These models allowed testing different setups and various radiofrequency protocols before an in-vivo study. Resulting qualitative and quantitative conclusions were helpful for the subsequent testing phase on a swine model.

The study included the application of the 3R principles as regards of Replacement of the animal model with an simulated mechanical model of 3D printed vertebra; Reduction of the total number of involved animals by modelization of the surgical access in multiple vertebrae and Refinement by identification of adequate surgical approaches in sedation followed by termination, without evaluation of the healing phase.

\section{Materials and Methods}

Simulated experiments were planned with several purposes: to perform an initial usability testing phase on the device, to collect R\&D data, to verify the instrument functionalities and to simulate different therapeutic treatment protocols. Distinct simulated models were selected to obtain as many significant results as possible before moving on to the later stage of the preclinical trial involving a swine model. A simple saline solution in contact with the RF needles of the device was used in order to assess the capability of the radiofrequency generator to induce heating through the agitation of free ions. Bovine liver slices, whose color immediately changes as a result of thermal ablation, were chosen as a simplified model to evaluate the twodimensional propagation of the radiofrequency field from the RF needle tip. Tests to control the size of the threedimensional thermal ablation zone were performed by immersing the RF needles in a Becker full of egg albumen. Lastly, a 3D printed vertebral replica filled with liver homogenate was selected as an in-vitro representative model to simulate both anatomy and impedance of the target tissue of the device (as suggested in a previous similar study). ${ }^{1}$ A further in-vivo test has then been carried out. One adult pig has been treated according to laboratory protocols. Fluoroscopy imaging was used to identify six target vertebrae to perform tumor ablation simulations with six different initial setups of the RF device on the same animal. Each vertebral body, previously identified with the corresponding treatment, has been explanted from the pig and stored in a fixative solution (formaldehyde at $10 \%$ ) for further histological investigations.

\section{Results}

The simple saline solution experiment allowed to properly ensure the correct functioning of the RF needles and the RF generator of the device. Bovine liver slices and egg albumen permitted to obtain crucial qualitative and quantitative results: shape of the ablated area-volume, characteristic time of the heating ramps, distal and proximal propagation of the radiofrequency field. Different device-related operating parameters have been monitored: impedance, thermocouple temperature, RF generator power. The same data have been collected by a more complex model: 3D printed vertebral replica filled with bovine liver homogenate.
Correspondence: Alice Ravizza, USE-ME-D srl I3P Politecnico di Torino, Torino, Italy.

E-mail: alice.ravizza@use-me-d.com

Key words: Vertebral models; spine tumor ablation; swine model.

Acknowledgments: FR: management of the project; AR: conceptualization and methodology. AR: resources. FR and AR: writingoriginal draft preparation. MM: supervision.

Disclosures: Use-me-d srl and Axon srl have mutual economic interest in the project.

Conference presentation: This paper was presented at the Third Centro 3R Annual Meeting - L'era delle 3R: modelli in silico, in vitro e in vivo per promuovere la ricerca traslazionale 30 September - 1 October 2021, Evento online organizzato dal Politecnico di Torino.

Received for publication: 9 July 2021.

Accepted for publication: 7 September 2021.

This work is licensed under a Creative Commons Attribution NonCommercial 4.0 License (CC BY-NC 4.0).

CC Copyright: the Author(s), 2021

Licensee PAGEPress, Italy

Biomedical Science and Engineering 2021; 4(s1):188 doi:10.4081/bse.2021.188

Simulation of ablation procedures on swine model allowed both procedural (RF electrodes placement, spinal access procedure, clinical reaction to the RF treatment) and post-procedural (shape of the ablated zone, presence of carbonization areas, histologic patterns in ablated vs nonablated tissue) evaluations, confirming results of previous in vitro tests. Simulated tumor ablation procedures have been performed on six different vertebrae of the animal.

\section{Discussion and Conclusions}

The preliminary set of functional tests on simulated models validated the pre-clinical operational assessment on the RF device. SPINERY is a complex and surgically invasive radiofrequency device whose functional evaluation would have required a substantial number of in vivo tests to get the same amount of information that has been obtained with the simplified yet representative simulated models. Bovine liver has been selected as a representative tissue of the metastatic spine tumor accordingly to the available literature, and it 


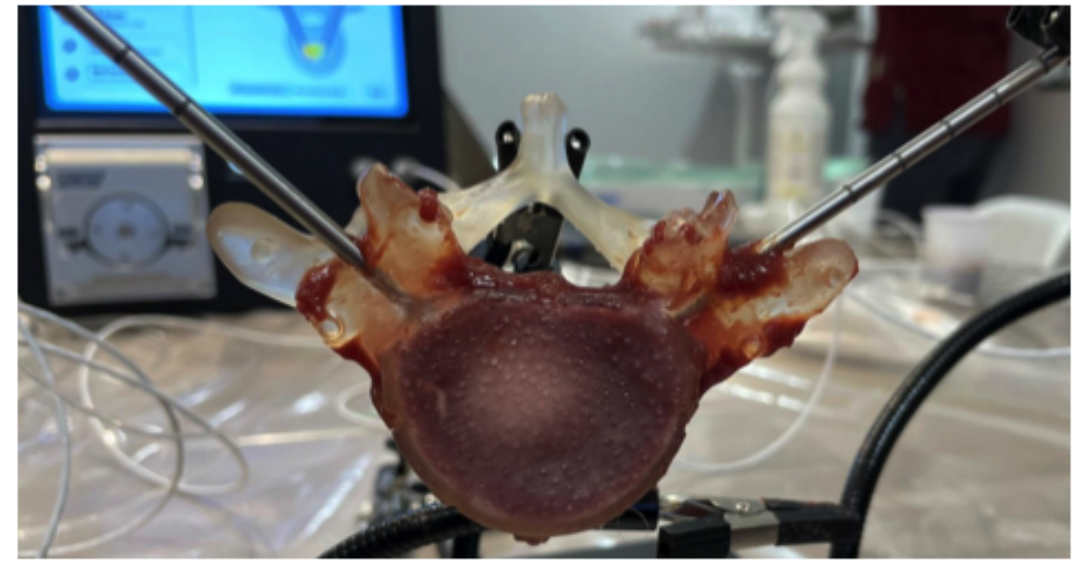

Figure 1. RF needles tested on a 3D-printed vertebra replica filled with liver homogenate.

has confirmed to be an effective alternative to collect qualitative and quantitative results, as well as a reasonable reference to monitor the thermal and electrical operating parameters of the radiofrequency device. The albumen-filled Becker showed to be a valid and immediate solution to easily conduct a large number of tests on controlling the size of the ablation volume through the cooling system of the RF needles. The relevance of the employed simulated models relies on the great amount of device-related functional data, and also on the proper planning of the final in vivo test, which limited the need for an animal trial to a single adult pig. SPINERY, includes different thermal ablation programs and a large number of RF needle configurations which may have required multiple animals. The results accomplished during the preliminary simulated studies allowed to select, amongst the potential initial setups of the device, a set of six thermal ablation scenarios which could be representative of the available treatment protocols offered by SPINERY. The significance of the obtained
Table 1. Ablation areas measured on six different target vertebrae.

\begin{tabular}{|l|l|l|}
\hline $\begin{array}{l}\text { Target } \\
\text { Vertebra }\end{array}$ & $\begin{array}{l}\text { Ablation } \\
\text { Area: } \\
\text { Transverse }\end{array}$ & $\begin{array}{l}\text { Ablation } \\
\text { Area: } \underline{\text { Sagittal }} \\
\text { Plane }\end{array}$ \\
$\begin{array}{l}\underline{\text { Plane }} \\
{\left[\mathbf{m m}^{2}\right]}\end{array}$ \\
\hline L4 & 106,27 & 106,18 \\
\hline L3 & 104,30 & 157,57 \\
\hline L2 & 70,63 & 93,82 \\
\hline L1 & 82,63 & 54,67 \\
\hline T12 & 136,42 & 48,37 \\
\hline T11 & 126,92 & 14,24 \\
\hline
\end{tabular}

results confirms the value of the chosen invitro models in reducing the need for an animal trial to test the device under examination.

\section{References}

1. Greenberg A, Weyel B, Sosna J, et al. The distribution of heat in bone during radiofrequency ablation of an ex vivo bovine model of osteoid osteoma. Bone Joint J 2014;96-B:677-83. 